\title{
Polycystic ovary syndrome is associated with increased osteopontin levels
}

\author{
Ali Saklamaz, Mehmet Calan', Ozgur Yilmaz², Tuncay Kume ${ }^{3}$, Muzaffer Temur², \\ Nurdan Yildiz ${ }^{4}$, Esin Kasap ${ }^{4}$, Mine Genc ${ }^{4}$, Banu Sarer Yurekli ${ }^{5}$ and \\ Gokcen Unal Kocabas ${ }^{1}$
}

Division of Endocrinology and Metabolism, Department of Internal Medicine, Sifa University School of Medicine, 35410 Izmir, Turkey, ${ }^{1}$ PCOS Research Group, Division of Endocrinology and Metabolism, Department of Internal Medicine, Izmir Bozyaka Training and Research Hospital, 35170 Bozyaka, Izmir, Turkey, ${ }^{2}$ PCOS Research Group, Department of Obstetrics and Gynecology, Manisa Merkezefendi State Hospital, 45020 Merkezefendi, Manisa, Turkey, ${ }^{3}$ Department of Biochemistry and Clinical Biochemistry, Dokuz Eylul University Faculty of Medicine, 35340 Inciralti, Izmir, Turkey, ${ }^{4}$ Department of Obstetrics and Gynecology, Sifa University School of Medicine, 35410 Izmir, Turkey and ${ }^{5}$ Division of Endocrinology and Metabolism, Department of Internal Medicine, Ege University Medical School, 35100 Bornova, Izmir, Turkey

Correspondence
should be addressed
to M Calan
Email
drmehmetcalan@gmail.com

\section{Abstract}

Objective: Osteopontin (OPN) is a multi-functional secreted glycoprotein that plays a crucial role in glucose metabolism and inflammatory process. Growing evidence suggests that there is a link between OPN and ovarian function. However, no such link has yet been found for OPN in polycystic ovary syndrome (PCOS). Our aim was to ascertain whether circulating OPN levels are altered in women with PCOS and to determine whether OPN levels differ between the follicular phase and mid-cycle of the menstrual cycle in eumenorrheic women.

Design and methods: In total, 150 women with PCOS and 150 age- and BMI-matched controls without PCOS were recruited for this prospective observational study. OPN levels were measured using ELISA. Metabolic parameters were also determined. Results: Circulating OPN levels were significantly elevated in PCOS women compared with controls $(69.12 \pm 31.59 \mathrm{ng} / \mathrm{ml}$ vs $42.66 \pm 21.28 \mathrm{ng} / \mathrm{ml} ; P<0.001)$. OPN levels were significantly higher at mid-cycle than in the follicular phase in eumenorrheic women. OPN was positively correlated with BMI, homeostasis model assessment of insulin resistance (HOMA-IR), free testosterone, and high sensitivity C-reactive protein (hs-CRP). Multivariate logistic regression analyses revealed that the odds ratio (OR) for PCOS was 3.64 for patients in the highest quartile of OPN compared with those in the lowest quartile $(\mathrm{OR}=3.64 ; 95 \% \mathrm{Cl}=2.42-5.57 ; P=0.011)$. Our findings indicate that $\mathrm{BMI}, \mathrm{HOMA}-\mathrm{IR}$, hs-CRP, and free testosterone are independent factors influencing serum OPN levels and that OPN is an independent predictor for HOMA-IR.

Conclusion: PCOS is associated with increased OPN levels.

\section{Introduction}

Polycystic ovary syndrome (PCOS) is a complex metabolic and reproductive disease. It is the most common endocrinopathy in women of reproductive age with a prevalence of $5-10 \%$. PCOS is characterized by ovulatory dysfunction, polycystic ovaries, biochemical (elevated androgens), and/or clinical (hirsutism and/or acne) (c) 2016 European Society of Endocrinology Printed in Great Britain hyperandrogenism. PCOS is an inflammatory-based metabolic disease associated with insulin resistance. Affected women present metabolic abnormalities including glucose intolerance, obesity, and dyslipidemia, as well as an increased risk of developing cardiovascular disease and type 2 diabetes $(1,2,3,4)$. Owing to the complexity of this

Published by Bioscientifica Ltd. 
disease, the causes of PCOS remain to be identified. In affected women, the amount of adipose tissue is increased, especially in the central area. Adipose tissue is an active endocrine organ secreting many cytokines (i.e. adipokines), which play a crucial role in the regulation of metabolic and reproductive functions as well as in the inflammatory response. Adipose tissue dysfunction exists in PCOS; this situation causes alteration of secreted adipokines and inflammatory mediators. In addition, disturbed secretion of adipokines contributes to the development of PCOS and also to metabolic abnormalities in the disease $(5,6)$.

Osteopontin (OPN) is a multi-functional secreted glycoprotein that plays a crucial role in a number of physiological and pathological events such as bone mineralization, atherosclerotic process, inflammation, insulin resistance, and malignant disorders. OPN is also a pro-inflammatory cytokine and an adhesion molecule which is expressed in various tissues including bone, bone marrow, vascular tissues, kidney, lungs, pancreas, ovary, and activated macrophages and T-lymphocytes $(7,8,9,10$, $11,12,13)$. It has been demonstrated that expression of OPN increases in adipose tissue in animal obesity model and obese subjects $(14,15)$. OPN induces macrophagesmonocytes recruitment in adipose tissue; therefore, OPN is implicated in the pathogenesis of obesity-induced adipose tissue inflammation and associated insulin resistance $(16,17,18)$. Increased concentrations of OPN have been observed in diseases related to chronic low-grade inflammation and insulin resistance such as obesity, diabetes, diabetic nephropathy, and cardiovascular diseases $(14,19,20,21)$.

There is also some evidence of a link between the OPN and ovarian function. Kuwabara et al. reported that OPN expression was stimulated in mouse ovaries by gonadotropin surge. They also observed that circulating OPN levels significantly increased following gonadotropin administration. They showed that OPN increases progesterone synthesis and expression of vascular endothelial growth factor (VEGF) in the early luteal phase (22). In addition, it has been previously demonstrated that circulating levels of certain adipokines show variations during the menstrual cycle $(23,24)$. Moreover, leptin surge (an adipokine) plays an active role in the onset of ovulation $(23,25)$. Therefore, in this study, we aimed to investigate: i) whether circulating OPN levels were altered in women with PCOS; ii) whether OPN is correlated with metabolic disturbances; and iii) whether OPN levels differed between the follicular phase and mid-cycle of the same ovulatory cycle in eumenorrheic women.

\section{Subjects and methods}

\section{Ethics statement}

This study was approved by the Local Ethics Committee of the Bozyaka Training and Research Hospital. The subjects gave their oral and written informed consent before their inclusion in the study. The study adhered strictly to the Declaration of Helsinki Principles as revised in 2008 .

\section{Subjects and study design}

The trial was designed as a prospective observational study and was conducted between January 2014 and December 2014 in the Department of Obstetrics and Gynecology of Merkezefendi State Hospital in Manisa, Turkey. We consecutively recruited 150 subjects with PCOS and 150 age- and BMI-matched subjects with normal menstrual cycles who presented to our clinic and met the inclusion and exclusion criteria. Forty healthy regularly menstruating women were randomly selected from the control group, and they followed mid-cycle (13-15 days) and mid-luteal phase (21-23 days) of the same menstrual cycle. We considered the cycle as ovulatory if a mid-cycle luteinizing hormone (LH) peak occurred and the mid-luteal serum progesterone exceeded a concentration of $10 \mathrm{ng} / \mathrm{ml}$ (26). All progesterone levels in these women were higher than $10 \mathrm{ng} / \mathrm{ml}$. Clinical and anthropometric variables were evaluated by a single investigator in all subjects. All the recruited women were Caucasian.

\section{PCOS group}

PCOS was diagnosed in this study based on the 2003 Rotterdam consensus criteria (27). However, we were more rigorous in the selection of patients, and only those who met all three of the following criteria were recruited for the PCOS group (after excluding other causes of hyperandrogenism and ovulatory dysfunction): i) oligo and/or anovulation; ii) clinical and/or biochemical signs of hyperandrogenism - the Ferriman-Gallwey (FG) method was used to determine hirsutism (28). Subjects were deemed hirsute if their FG score was $\geq 8$. Biochemical hyperandrogenism was defined as an increase in the serum concentrations of free testosterone (normal values: $2.9-31.8 \mathrm{ng} / \mathrm{dl}$ ) and/or total testosterone (normal values: $0.15-0.7 \mathrm{ng} / \mathrm{ml}$ ), and/or a DHEAS (normal values: $10-248 \mu \mathrm{g} / \mathrm{dl}$ ); and iii) typical ultrasonographic finding of polycystic ovaries (with one ovary being sufficient for diagnosis), defined as the 
presence of $\geq 12$ follicles measuring $2-9 \mathrm{~mm}$ in diameter or an ovarian volume of $>10 \mathrm{ml}$ (without a cyst or dominant follicle in either ovary).

\section{Control group}

Control subjects were recruited from healthy women who visited the gynecology or endocrinology clinic for routine examination or from hospital workers and university students by means of an advertisement at the university. All volunteers for the control group had regular menstrual cycles, and they had no concomitant health problems or signs of hirsutism or acne or hyperandrogenism.

\section{Exclusion criteria}

The exclusion criteria adopted during subject selection were: i) other causes of irregular menstrual cycles and/or androgen excess (i.e. Cushing's syndrome; hyperprolactinemia; congenital adrenal hyperplasia; or other diseases of the adrenal gland, thyroid disorders, galactorrhea, breastfeeding, and pregnancy); ii) impaired glucose tolerance or type $1 / 2$ diabetes; 3 ) hypertension, hyperlipidemia, active or chronic liver or renal failure, or congestive heart failure; iv) a history of coronary artery disease, gestational diabetes mellitus (GDM), or acute infection (within the preceding 14 days); v) presence of any chronic inflammatory and autoimmune disease; vi) known malignancy; vii) hormonal contraception and/or anti-androgen therapy (within the preceding 6 months); and viii) BMI $<18.5$ or $\geq 35 \mathrm{~kg} / \mathrm{m}^{2}$, age $<18$ or $>35$ years. The use of medications for dyslipidemia, hypertension, hyperglycemia, insulin resistance, or obesity was an exclusion criterion for these studies. None of the subjects used tobacco or alcohol.

\section{Anthropometric evaluation}

Of the individuals included in the study, a detailed history was taken. Following general physical examination of the subjects, anthropometric measurements were performed (age, weight, height, and waist circumference). Height $(\mathrm{cm})$ and weight $(\mathrm{kg})$ were measured with the subject barefoot in light daily clothes. BMI was calculated using the formula weight $(\mathrm{kg}) / \mathrm{square}$ meter of height $\left(\mathrm{m}^{2}\right)$. Waist circumference $(\mathrm{cm})$ was measured midway the lower rib margin and the iliac crest at the end of a gentle expiration. Blood pressure was measured in the sitting position after a rest period of at least $15 \mathrm{~min}$. The average of three measurements was obtained.

\section{Biochemical evaluation}

Venous blood samples were collected from the antecubital veins of the subjects during the early follicular phase of menstrual bleeding (3rd-5th days) in the morning (between 0800-0900 h), after the onset of early spontaneous or progesterone-induced menses, following at least $12 \mathrm{~h}$ of fasting. Samples were separated by centrifugation for $15 \mathrm{~min}$ at $2000 \boldsymbol{g}$ after clotting for $30 \mathrm{~min}$ at room temperature. The serum samples were subsequently stored in aliquots at $-80{ }^{\circ} \mathrm{C}$ prior to the analysis of OPN. Furthermore, 40 women in the control group followed mid-cycle and in the luteal phase of the same menstrual cycle. Following at least 12-h fasting, blood was drawn in days 13-15 (ovulation) of the menstrual cycle to measure OPN and LH levels. Also, blood was drawn in days 21-23 (mid-luteal phase) of the menstrual cycle to measure progesterone levels.

Fasting blood glucose (FBG), serum insulin, high sensitivity C-reactive protein (hs-CRP), total cholesterol, triglycerides, HDL cholesterol (HDL-C), LDL cholesterol (LDL-C), free testosterone, total testosterone, DHEAS, follicle-stimulating hormone ( $\mathrm{FSH}), \mathrm{LH}$, estradiol $\left(\mathrm{E}_{2}\right)$, and OPN levels were measured.

FBG, serum hs-CRP, total cholesterol, total HDL-C, and triglyceride levels were determined using an autoanalyzer (Abbott Architect C 16000) with its dedicated kits (Abbott Diagnostics). LDL-C was calculated using the Friedewald equation: LDL-C $=$ total cholesterol $-(\mathrm{HDL}-$ $\mathrm{C}+$ triglyceride/5). Serum insulin levels were measured by an auto-analyzer (Abbott Architect I2000) using chemiluminescent microparticle immunoassay (CMIA) with its dedicated kits (Abbott Diagnostics). Serum FSH, LH, $\mathrm{E}_{2}$, total testosterone, and DHEAS levels were measured by CMIA technique (Beckman Coulter, Inc., Brea, CA, USA). Serum free testosterone levels were measured using the RIA method (DSL 4900 Gamma Counter, Beckman Coulter, Inc., Fullerton, CA, USA). Insulin resistance was calculated for each participant using the homeostasis model assessment of insulin resistance $($ HOMA-IR $)=$ fasting insulin $(\mu \mathrm{U} / \mathrm{ml}) \times$ fasting glucose $(\mathrm{mg} / \mathrm{dl}) / 405$ (29).

\section{OPN ELISA}

Serum OPN levels (Cusabio Biotech, Hubei, China) were measured using commercially available ELISA kits. The intra-assay coefficient of variation (CV) was $<6.1 \%$ and the inter-assay $\mathrm{CV}<6.7 \%$. All samples were analyzed in duplicate. The assays have high sensitivity and excellent 
specificity for the detection of human OPN with no significant cross-reactivity or interference.

\section{Statistical analysis}

Power analysis $>$ The power analysis was performed using G Power 3.0.10 for Windows. According to our pilot study results for circulating OPN levels, the required size of the study population was calculated to be 114 subjects/group $(\alpha=0.05$ and the study power $=0.90)$.

Normal distribution of the data was tested using the Kolmogorov-Smirnov test. The skewness of the distribution required logarithmic transformation for statistical analysis of serum DHEAS. Continuous variables were presented as mean \pm s.D. Demographic and laboratory characteristics of the studied women with and without PCOS were compared using independent samples $t$-test (two-tailed). Differences in follicular phase and mid-cycle OPN levels in eumenorrheic women were analyzed by a paired $t$-test. Subjects were divided into two groups according to their BMI levels (BMI $<25$ and $\geq 25 \mathrm{~kg} / \mathrm{m}^{2}$ ). We compared subjects according to their BMI in PCOS and control group using independent samples $t$-test (twotailed). We divided PCOS subjects into two groups according to the presence of insulin resistance (HOMAIR $>2.71$ and $\leq 2.71$ ). We compared serum OPN levels in the groups using independent samples $t$-test (two-tailed). The relation of OPN with other demographic and laboratory characteristics was evaluated using Pearson's correlation analysis. Subjects were divided into four groups according to their OPN levels. To examine the association of OPN levels (quartiles) and the development of PCOS, odds ratio (OR) was calculated with multivariate logistic regression analysis. The model was adjusted for potential confounders (i.e. age, BMI, HOMA-IR, free testosterone, hs-CRP, and lipids). Model compatibility was analyzed using the Hosmer and Lemeshow test in all cases, and the analysis revealed that the models were compatible $(P>0.05)$. To identify independent relationships between OPN levels and age, BMI, HOMA-IR, free testosterone, hs-CRP, and triglycerides multiple linear regression analyses were performed. To identify independent relationship between HOMA-IR and OPN, multiple linear regression analyses were performed (age, BMI, hs-CRP, lipids, and free testosterone were consecutively added in the analysis). In both multiple regression analyses, PCOS status was added for adjusting in models as a covariate. All independent variables in the multiple linear regression were tested for multicollinearity. If the variance inflation factor exceeded 2.5 , the variable was considered to be collinear. Therefore, waist circumference, FBG, insulin, and total testosterone were not included in the models.

All reported CI values are calculated at the 95\% level. A two-sided $P$ value $<0.05$ was considered statistically significant. All analyses were performed using the Statistical Package for the Social Sciences Software version 18.0 (SPSS, Inc.).

\section{Results}

\section{Clinical and laboratory characteristics of the study population}

The comparative demographic and laboratory parameters of the studied women with and without PCOS are given in Table 1.

As subjects were matched for age and BMI, both parameters were similar in between the two groups $(P>0.05)$.

Circulating OPN levels were significantly elevated in women with PCOS compared with controls (69.12 \pm $31.59 \mathrm{ng} / \mathrm{ml}$ vs $42.66 \pm 21.28 \mathrm{ng} / \mathrm{ml} ; P<0.001$; Fig. 1 A).

Table 1 Comparison of the demographic and laboratory characteristics of the subjects. Results are given in mean \pm s.D.

\begin{tabular}{|c|c|c|c|}
\hline Variables & $\begin{array}{l}\text { PCOS } \\
(n=150)\end{array}$ & $\begin{array}{l}\text { Controls } \\
(n=150)\end{array}$ & $\boldsymbol{P}^{*}$ \\
\hline Age (years) & $27.78 \pm 3.94$ & $27.67 \pm 3.54$ & 0.794 \\
\hline BMI $\left(\mathrm{kg} / \mathrm{m}^{2}\right)$ & $26.73 \pm 3.46$ & $26.33 \pm 3.44$ & 0.308 \\
\hline $\begin{array}{l}\text { Waist circumference } \\
(\mathrm{cm})\end{array}$ & $87.17 \pm 8.90$ & $85.56 \pm 9.34$ & 0.127 \\
\hline SBP $(\mathrm{mmHg})$ & $111.60 \pm 10.48$ & $110.46 \pm 11.70$ & 0.378 \\
\hline $\mathrm{DBP}(\mathrm{mmHg})$ & $70.86 \pm 6.15$ & $0 \pm 6.97$ & 0.255 \\
\hline $\begin{array}{l}\text { Ferriman-Gallwey } \\
\text { score }\end{array}$ & $12.01 \pm 2.79$ & $4.26 \pm 1.24$ & $<0.001 *$ \\
\hline $\mathrm{FBG}(\mathrm{mg} / \mathrm{dl})$ & $88.29 \pm 5.99$ & $83.72 \pm 7.12$ & $<0.001$ * \\
\hline Insulin $(\mu \mathrm{lU} / \mathrm{ml})$ & $13.56 \pm 3.65$ & $8.83 \pm 3.18$ & $<0.001^{*}$ \\
\hline HOMA-IR & $2.97 \pm 0.87$ & $1.82 \pm 0.68$ & $<0.001^{*}$ \\
\hline $\begin{array}{l}\text { Total cholesterol } \\
\quad(\mathrm{mg} / \mathrm{dl})\end{array}$ & $208.05 \pm 40.18$ & $192.41 \pm 29.62$ & $<0.001 *$ \\
\hline LDL-C (mg/dl) & $133.96 \pm 36.42$ & $123.57 \pm 26.56$ & $0.005^{*}$ \\
\hline HDL-C (mg/dl) & $50.12 \pm 11.84$ & $51.70 \pm 10.97$ & 0.231 \\
\hline Triglycerides (mg/dl) & $135.56 \pm 42.75$ & $85.96 \pm 37.26$ & $<0.001 *$ \\
\hline hs-CRP (mg/l) & $1.45 \pm 0.52$ & $0.57 \pm 0.28$ & $<0.001^{*}$ \\
\hline $\mathrm{FSH}(\mathrm{mlU} / \mathrm{ml})$ & $5.95 \pm 0.94$ & $6.11 \pm 1.20$ & 0.182 \\
\hline $\mathrm{LH}(\mathrm{mlU} / \mathrm{ml})$ & $9.76 \pm 2.40$ & $5.11 \pm 1.35$ & $<0.001 *$ \\
\hline$E_{2}(p g / m l)$ & $49.09 \pm 9.66$ & $47.20 \pm 8.96$ & 0.080 \\
\hline $\begin{array}{l}\text { Free testosterone, } \\
\quad(\mathrm{ng} / \mathrm{dl})\end{array}$ & $52.45 \pm 12.44$ & $14.35 \pm 3.25$ & $<0.001^{*}$ \\
\hline $\begin{array}{l}\text { Total testosterone } \\
(\mathrm{ng} / \mathrm{ml})\end{array}$ & $0.42 \pm 0.12$ & $0.39 \pm 0.11$ & $0.018 *$ \\
\hline DHEAS $(\mu \mathrm{g} / \mathrm{dl})$ & $149.77 \pm 35.87$ & $141.37 \pm 40.07$ & 0.057 \\
\hline
\end{tabular}

SBP, systolic blood pressure. Independent samples $t$-test was used. A $P$ value of $<0.05$ was considered significant $\left(^{*}\right)$. 

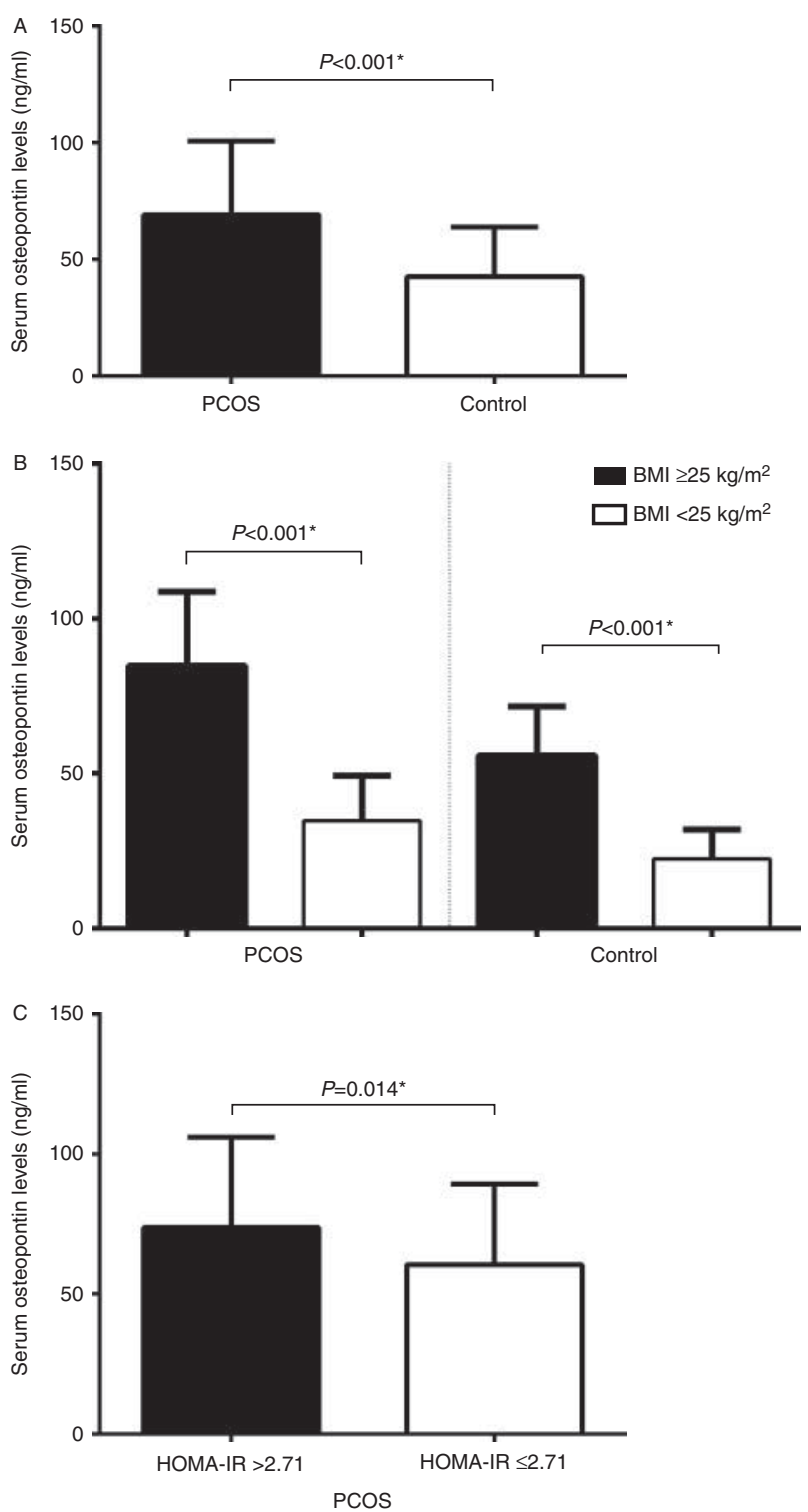

Figure 1

Circulating osteopontin (OPN) levels in study population.

(A) Circulating OPN levels in the control and PCOS groups.

(B) Circulating OPN levels according to BMI (normal weight: BMI $<25 \mathrm{~kg} / \mathrm{m}^{2}$ and overweight/obese: BMI $\geq 25 \mathrm{~kg} / \mathrm{m}^{2}$ ).

(C) Circulating OPN levels in PCOS women with and without insulin resistance (HOMA-IR $>2.71$ insulin resistant and HOMA-IR $\leq 2.71$ normal).

FBG, serum insulin, hs-CRP, and HOMA-IR levels were found to be increased in women with PCOS. Furthermore, serum total cholesterol, LDL-C, and triglycerides levels are significantly higher in PCOS subjects than in controls. Serum free testosterone and total testosterone levels were significantly higher in women with PCOS. We stratified recruited subjects into two groups according to their BMI levels $\left(<25\right.$ and $\left.\geq 25 \mathrm{~kg} / \mathrm{m}^{2}\right)$. For the control group, there were 59 subjects with BMI $<25 \mathrm{~kg} / \mathrm{m}^{2}$ and 91 subjects with BMI $\geq 25 \mathrm{~kg} / \mathrm{m}^{2}$; for the PCOS group, there were 47 patients with BMI $<25 \mathrm{~kg} / \mathrm{m}^{2}$ and 103 patients with BMI $\geq 25 \mathrm{~kg} / \mathrm{m}^{2}$. We compared OPN levels in each group according to their BMI. In terms of BMI, there was no significant difference in the number of subjects in between the groups $(P=0.184$; data not shown). As shown in Fig. 1B, overweight/obese women (BMI $\geq 25 \mathrm{~kg} / \mathrm{m}^{2}$ ) had significantly higher circulating OPN levels than lean subjects both in control and PCOS groups $\left(\mathrm{BMI}<25 \mathrm{~kg} / \mathrm{m}^{2}\right)$.

We divided PCOS patients into two groups as being insulin resistant or not (HOMA-IR > 2.71 and $\leq 2.71)$ (30). Out of 150 subjects, 98 were diagnosed as being insulin resistant. As shown in Fig. 1C, PCOS patients with insulin resistance had significantly elevated circulating OPN levels compared to those without insulin resistance $(P=0.014)$.

\section{Comparison of follicular and mid-cycle serum OPN levels in healthy eumenorrheic women}

Differences in follicular phase and mid-cycle Müllerianinhibiting factor levels in the eumenorrheic women were analyzed by a paired $t$-test. Circulating OPN levels were higher at mid-cycle compared to the follicular phase (follicular phase: $40.82 \pm 18.99 \mathrm{ng} / \mathrm{ml}$ and mid-cycle: $44.90 \pm 20.53 \mathrm{ng} / \mathrm{ml} ; P=0.001)$. The results are shown as a box plot graph (Fig. 2).

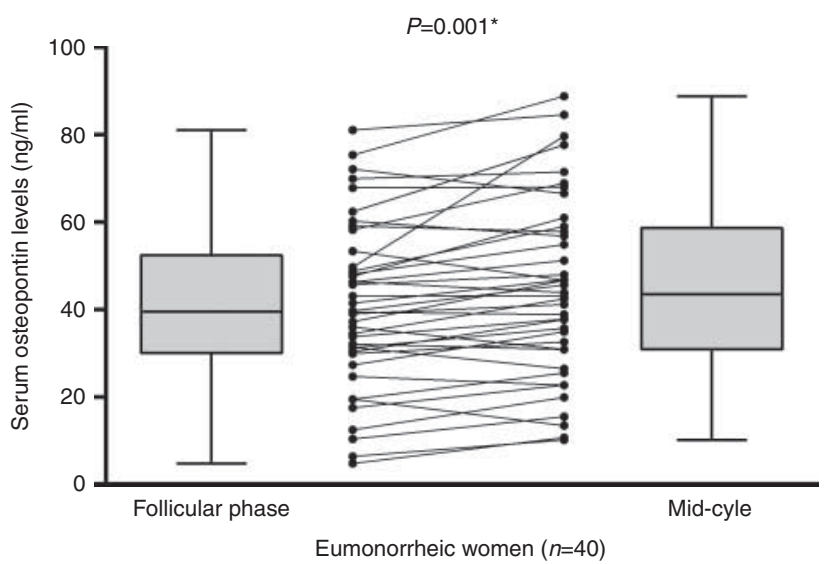

Figure 2

Box plot for serum osteopontin levels in follicular and mid-cycle in eumenorrheic women. 
Table 2 Correlation coefficient between OPN levels and clinical parameters.

\begin{tabular}{|c|c|c|c|c|}
\hline & \multicolumn{4}{|c|}{ OPN } \\
\hline & \multicolumn{2}{|c|}{ PCOS } & \multicolumn{2}{|c|}{ Control } \\
\hline & $r$ & $P$ & $r$ & $P$ \\
\hline Age & 0.118 & 0.245 & 0.103 & 0.136 \\
\hline $\mathrm{BMI}$ & 0.245 & $0.029 *$ & 0.168 & $0.034 *$ \\
\hline Waist circumference & 0.284 & $0.015^{*}$ & 0.175 & $0.039 *$ \\
\hline $\begin{array}{l}\text { Systolic blood } \\
\text { pressure }\end{array}$ & 0.117 & 0.249 & 0.125 & 0.643 \\
\hline $\begin{array}{l}\text { Diastolic blood } \\
\text { pressure }\end{array}$ & 0.131 & 0.431 & 0.105 & 0.461 \\
\hline Insulin & 0.341 & $0.004^{*}$ & 0.137 & $0.016 *$ \\
\hline FBG & 0.137 & $0.019 *$ & 0.115 & $0.025^{*}$ \\
\hline HOMA-IR & 0.298 & $0.012^{*}$ & 0.126 & $0.021 *$ \\
\hline Free testosterone & 0.317 & $<0.001 *$ & 0.147 & 0.265 \\
\hline Total testosterone & 0.122 & 0.068 & 0.114 & 0.213 \\
\hline hs-CRP & 0.361 & $<0.001 *$ & 0.138 & $0.029 *$ \\
\hline Total cholesterol & 0.127 & 0.085 & 0.106 & 0.133 \\
\hline LDL-C & 0.166 & 0.134 & 0.114 & 0.109 \\
\hline HDL-C & -0.151 & 0.078 & 0.106 & 0.127 \\
\hline Triglycerides & 0.231 & $0.037 *$ & 0.131 & 0.056 \\
\hline
\end{tabular}

Pearson's correlation analysis was used. $r$, Pearson's correlation coefficient. A $P$ value of $<0.05$ was considered significant $(*)$.

\section{Correlation of OPN with clinical parameters}

We next investigated the relationship between circulating OPN levels and various other parameters in women with PCOS. Correlation coefficients are shown in Table 2.

Circulating OPN levels were positively correlated with BMI, waist circumference, insulin, FBG, HOMA-IR, and hs-CRP in both groups. In addition, there was a positive correlation between OPN and free testosterone, triglycerides in only PCOS women. There was no significant correlation between OPN levels and age, blood pressure, and other lipids profile.

\section{Multivariate binary logistic regression analysis}

The independent association between PCOS and OPN levels (quartiles) was assessed by multivariate logistic regression analysis for the potential confounders including age, BMI, HOMA-IR, free testosterone, hs-CRP, and lipids (Fig. 3).

Multivariate logistic regression analyses revealed that the OR for PCOS was 3.64 for patients in the highest quartile of OPN compared with those in the lowest quartile $(\mathrm{OR}=3.64 ; 95 \% \mathrm{CI}=2.42-5.57 ; P=0.011)$, after potential confounders. Odds of PCOS did not differ between subjects with the third $(\mathrm{OR}=1.53 ; 95 \%$ $\mathrm{CI}=0.89-2.63, \quad P=0.089)$, second $\quad(\mathrm{OR}=1.21 ; 95 \%$ $\mathrm{CI}=0.71-2.06 ; P=0.186)$, and the lowest quartile OPN.

\section{Multivariate regression analysis}

To verify independent associations between OPN and metabolic profile, multiple linear regression analyses were performed (Table 3). This model explained $41.2 \%$ of the total variability in OPN concentration. Multiple linear regression analysis revealed that BMI, HOMA-IR, hs-CRP, and free testosterone independently predicted OPN levels.

To verify independent associations between OPN and HOMA-IR, multiple linear regression analysis was performed (Table 4). In unadjusted analysis, OPN predicted degree of insulin resistance. To clarify the effects of OPN on HOMA-IR, age, BMI, hs-CRP, lipids, and free testosterone were consecutively added in the analysis as potential confounders. After adjustment for these parameters, OPN levels remained an independent predictor for HOMA-IR.

\section{Discussion}

Growing evidence suggests that OPN plays a role in the reproductive system of women. This is, to our knowledge, the first report describing the circulating OPN levels in PCOS patients. In the present study, we have found that circulating OPN levels were significantly higher in PCOS compared with controls, and also circulating OPN levels vary throughout the menstrual cycle in healthy eumenorrheic women, being higher at mid-cycle than in the

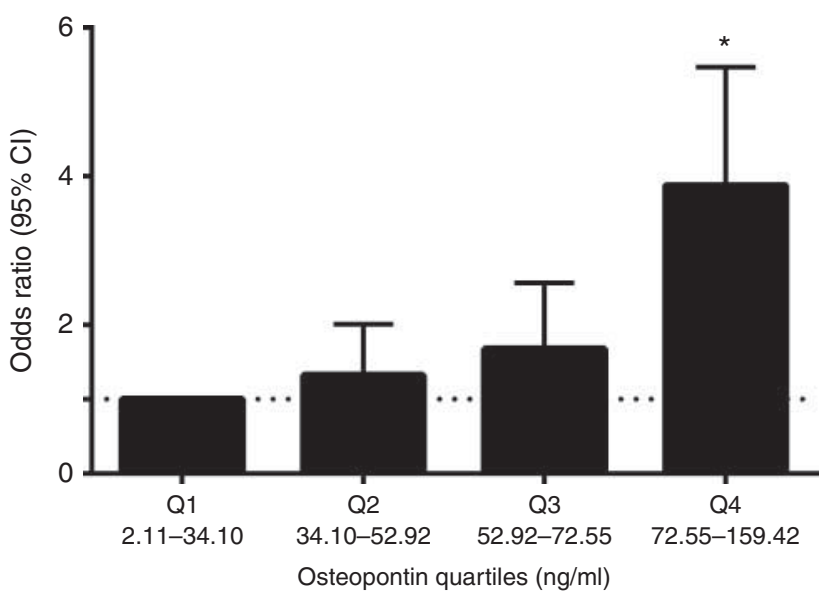

\section{Figure 3}

Association of osteopontin (OPN) with PCOS in adjusted models. Multivariate adjusted OR for having PCOS according to the quartiles of OPN (reference, the lowest quartile). Model adjusted for age, BMI, HOMA-IR, testosterones, hs-CRP, and lipids. A $P$ value of $<0.05$ was considered significant $(*)$. OR, odds ratio; $Q$, quartile. 
Table 3 Evaluation of the effects of age, BMI, HOMA-IR, free testosterone, hs-CRP, and triglycerides on OPN in whole study population using the multiple linear regression analysis $\left(R^{2}=0.412\right)$.

\begin{tabular}{|c|c|c|c|c|}
\hline & \multirow[b]{2}{*}{$\beta$} & \multicolumn{2}{|c|}{$95 \% \mathrm{Cl}$} & \multirow[b]{2}{*}{$\boldsymbol{P}$} \\
\hline & & Lower & Upper & \\
\hline \multicolumn{5}{|l|}{ Variables } \\
\hline Age & 0.164 & -0.730 & 1.058 & 0.298 \\
\hline BMI & 1.583 & 0.551 & 2.615 & $0.024 *$ \\
\hline HOMA-IR & 2.185 & 0.199 & 4.171 & $0.011 *$ \\
\hline Free testosterone & 1.459 & 0.347 & 2.571 & $0.021 *$ \\
\hline hs-CRP & 1.907 & 0.239 & 3.575 & $0.015^{*}$ \\
\hline Triglycerides & 0.114 & -0.031 & 0.259 & 0.371 \\
\hline
\end{tabular}

Multiple linear regression analysis was used. $\beta$, unstandardized regression coefficient. A $P$ value of $<0.05$ was considered significant $\left(^{*}\right)$.

follicular phase. Importantly, overweight/obese women also had significantly higher circulating OPN levels than lean women regardless of PCOS. PCOS patients with insulin resistance had significantly elevated circulating OPN levels compared with those without insulin resistance. Logistic regression analysis revealed that increased circulating OPN levels were significantly associated with high odds of having PCOS. Subjects with the highest quartile OPN levels showed a 3.64 times increased risk for having PCOS, compared to those in the lowest quartile after adjusting for potential confounders. In addition, multivariate regression analyses showed that BMI, HOMAIR, hs-CRP, and free testosterone were independent factors influencing serum OPN levels.

OPN is a multifunctional pro-inflammatory cytokine that is able to promote inflammation and insulin resistance in adipose tissues. Increased concentrations of OPN have been observed in diseases related to chronic low-grade inflammation and insulin resistance $(16,17,18)$. OPN levels were found to be elevated in type 2 diabetes mellitus compared with controls. OPN was positively correlated with FBG whereas it was inversely correlated with insulin sensitivity (20). Gómez-Ambrosi et al. reported that circulating OPN levels were elevated in overweight and obese subjects; furthermore, OPN concentration was correlated with body fat. In addition, they reported that OPN levels significantly decreased following weight loss (14). PCOS is an inflammatory-based metabolic disease associated with insulin resistance $(1,2,3)$. In the present study, we found that circulating OPN levels were significantly elevated in women with PCOS compared with controls, and also we found a positive correlation between OPN and insulin resistance marker HOMA-IR. Our findings further indicated that OPN was an independent predictor of HOMA-IR. Moreover, insulinresistant PCOS subjects had elevated OPN levels compared with PCOS subjects without insulin resistance. Many women with PCOS have some degree of insulin resistance (5). The pathogenic mechanisms involved in the increased insulin resistance associated with PCOS is still poorly understood, but accumulating evidence highlights the potential role of chronic low-grade inflammation as one of the main contributors (5). We can speculate that increased OPN levels may contribute to the development of insulin resistance in women with PCOS. On the other hand, contradictory results have been reported about OPN levels in GDM. In a study, OPN levels were found to be decreased in GDM compared with control and did not correlate with insulin sensitivity, whereas another study reported that OPN levels were not different between two groups $(31,32)$.

Although the underlying pathophysiologic mechanisms have not yet been determined clearly, adipose tissue dysfunction exists in PCOS $(5,6)$. Pro-inflammatory immune cell accumulation in adipose tissue is associated with the development of insulin resistance. OPN induces both macrophage recruitment in adipose tissue and secretion of cytokines. OPN plays a crucial role in the development of adipose tissue inflammation and insulin resistance $(16,17,18,33)$. Increased expression of OPN has been reported in obese mice and obese humans $(14,15)$. Adipose tissue dysfunction may also impact the pathophysiology of PCOS through its influence on metabolism and reproductive systems (6). In the present study, circulating OPN levels were significantly elevated in women with PCOS, and also overweight/obese women had significantly higher circulating OPN levels than lean

Table 4 Evaluation of the effects of OPN, age, BMI, hs-CRP, lipids, and free testosterone on HOMA-IR in whole study population using the multiple linear regression analysis.

\begin{tabular}{|c|c|c|c|c|}
\hline & \multirow[b]{2}{*}{$\beta$} & \multicolumn{2}{|c|}{$95 \% \mathrm{Cl}$} & \multirow[b]{2}{*}{$\boldsymbol{P}$} \\
\hline & & Lower & Upper & \\
\hline \multicolumn{5}{|l|}{ Variables } \\
\hline Osteopontin & 0.289 & 0.085 & 0.493 & $0.004 *$ \\
\hline Adjusted for age + BMI & 0.285 & 0.061 & 0.509 & $0.006 *$ \\
\hline $\begin{array}{l}\text { Adjusted for age }+ \\
\text { BMI + hs-CRP }\end{array}$ & 0.281 & 0.024 & 0.538 & $0.006^{*}$ \\
\hline $\begin{array}{l}\text { Adjusted for age }+ \\
\mathrm{BMI}+\mathrm{hs}-\mathrm{CRP}+\text { lipids }\end{array}$ & 0.279 & 0.016 & 0.542 & 0.007 * \\
\hline $\begin{array}{l}\text { Adjusted for age }+ \\
\text { BMI }+ \text { hs-CRP-lipids }+ \\
\text { free testestrone }\end{array}$ & 0.203 & 0.024 & 0.382 & $0.013^{*}$ \\
\hline
\end{tabular}

Multiple linear regression analysis was used. $\beta$, unstandardized regression coefficient. A $P$ value of $<0.05$ was considered significant (*). 
women regardless of PCOS status. Circulating OPN levels were correlated with BMI and waist circumference. Moreover, multivariate logistic regression analyses revealed that increased OPN levels were significantly associated with high odds of having PCOS after adjustment for potential confounders. Although the physiological roles of OPN in the ovulation system have not been elucidated clearly yet, based on these observations, it is reasonable to speculate that OPN may contribute to the development of PCOS independently. In addition, OPN may contribute to the metabolic disturbances in PCOS patients. Nevertheless, the underlying mechanisms explaining the present results require further investigation.

Kuwabara et al. (22) recently reported that OPN might be involved in the regulation of follicular growth and ovulation. They showed that OPN expression increased in mouse ovarian granulosa cells in response to gonadotropin. They demonstrated that OPN increased progesterone synthesis and expression of angiogenic factor, VEGF. In addition, they observed circulating levels of OPN increased following gonadotropin administration. Consistent with this study, we found that circulating OPN levels vary throughout the menstrual cycle in healthy eumenorrheic women, being higher at mid-cycle than in the follicular phase. In light of these data, OPN might play a role in the ovulation. Taken together, increased circulating OPN levels in PCOS may implicate a possible other mechanism: there is a defect on OPN-mediated signaling pathway; therefore, secretion of OPN increases to overcome this problem in PCOS subjects.

There are some limitations to the current study. Although our sample size is relatively large, the crosssectional design of the study cannot prove causality. Another limitation the study has is the technique we used to evaluate insulin resistance. We used the less invasive HOMA-IR method, although it is inferior to the clamp technique. Lastly, we measured free testosterone levels using radioimmuno assay methods to determine androgen excess, although it is less reliable than free androgen index in women.

In conclusion, our results reveal elevated OPN levels in PCOS. However, it is not clear whether increased OPN levels are primary activators or they simply result from the disorder. In addition, OPN levels were significantly higher at mid-cycle than in the follicular phase in eumenorrheic women. More importantly, increased OPN levels were significantly associated with high odds of having PCOS. OPN may play a crucial role in the female reproductive system, including the development of PCOS and normal ovulation in humans. The physiological and pathological significance of our findings remains to be further elucidated.

\section{Declaration of interest}

The authors declare that there is no conflict of interest that could be perceived as prejudicing the impartiality of the research reported.

\section{Funding}

This research did not receive any specific grant from any funding agency in the public, commercial or not-for-profit sector.

\section{Author contribution statement}

A Saklamaz, M Calan, O Yilmaz, T Kume, M Temur, N Yildiz, and E Kasap participated in study design and performed ELISA. M Calan, M Genc, G Unal Kocabas, and A Saklamaz participated in study design, analyzed the data, wrote, reviewed, and edited the manuscript. O Yilmaz and M Temur provided serum samples and contributed to discussions of data interpretation. B Sarer Yurekli reviewed and edited the manuscript, contributed to discussions. M Calan is the guarantor of this work and, as such, had full access to all the data in the study and takes responsibility for the integrity of the data and the accuracy of the data analysis.

\section{References}

1 Ehrmann DA. Polycystic ovary syndrome. New England Journal of Medicine 2005352 1223-1236. (doi:10.1056/ NEJMra041536)

2 Azziz R, Woods KS, Reyna R, Key TJ, Knochenhauer ES \& Yildiz BO. The prevalence and features of the polycystic ovary syndrome in an unselected population. Journal of Clinical Endocrinology and Metabolism 200489 2745-2749. (doi:10.1210/jc.2003-032046)

3 Legro RS, Arslanian SA, Ehrmann DA, Hoeger KM, Murad MH, Pasquali R \& Welt CK. Diagnosis and treatment of polycystic ovary syndrome: an Endocrine Society clinical practice guideline. Journal of Clinical Endocrinology and Metabolism 201398 4565-4592. (doi:10.1210/ jc.2013-2350)

4 Jayasena CN \& Franks S. The management of patients with polycystic ovary syndrome. Nature Reviews. Endocrinology 201410 624-636.

5 Diamanti-Kandarakis E \& Dunaif A. Insulin resistance and the polycystic ovary syndrome revisited: an update on mechanisms and implications. Endocrine Reviews 201233 981-1030. (doi:10.1210/er. 2011-1034)

6 Spritzer PM, Lecke SB, Satler F \& Morsch DM. Adipose tissue dysfunction, adipokines, and low-grade chronic inflammation in polycystic ovary syndrome. Reproduction 2015149 R219-R227. (doi:10.1530/REP-14-0435)

7 Weber GF \& Cantor H. The immunology of Eta-1/osteopontin. Cytokine \& Growth Factor Reviews 19967 241-248. (doi:10.1016/ S1359-6101(96)00030-5)

8 Standal T, Borset M \& Sundan A. Role of osteopontin in adhesion, migration, cell survival and bone remodeling. Experimental Oncology 200426 179-184.

9 Mazzali M, Kipari T, Ophascharoensuk V, Wesson JA, Johnson R \& Hughes J. Osteopontin - a molecule for all seasons. QJM: Monthly Journal of the Association of Physicians 200295 3-13. (doi:10.1093/qjmed/95.1.3)

10 Subramani V. OPN-revisited. Journal of Clinical and Diagnostic Research 20159 ZE10-ZE13. (doi:10.7860/JCDR/2015/12872.6111) 
11 Evrard S, Delanaye P, Kamel S, Cristol J-P \& Cavalier E. Vascular calcification: from pathophysiology to biomarkers. Clinica Chimica Acta 2015438 401-414. (doi:10.1016/j.cca.2014.08.034)

12 Brown LF, Berse B, Van de Water L, Papadopoulos-Sergiou A, Perruzzi CA, Manseau EJ, Dvorak HF \& Senger DR. Expression and distribution of osteopontin in human tissues: widespread association with luminal epithelial surfaces. Molecular Biology of the Cell 19923 1169-1180. (doi:10.1091/mbc.3.10.1169)

13 Scatena M, Liaw L \& Giachelli CM. Osteopontin: a multifunctional molecule regulating chronic inflammation and vascular disease. Arteriosclerosis, Thrombosis, and Vascular Biology 200727 2302-2309. (doi:10.1161/ATVBAHA.107.144824)

14 Gómez-Ambrosi J, Catalán V, Ramírez B, Rodríguez A, Colina I, Silva C, Rotellar F, Mugueta C, Gil MJ, Cienfuegos JA et al. Plasma osteopontin levels and expression in adipose tissue are increased in obesity. Journal of Clinical Endocrinology and Metabolism 200792 3719-3727. (doi:10.1210/jc.2007-0349)

15 Kiefer FW, Zeyda M, Todoric J, Huber J, Geyeregger R, Weichhart T, Aszmann O, Ludvik B, Silberhumer GR, Prager G et al. Osteopontin expression in human and murine obesity: extensive local up-regulation in adipose tissue but minimal systemic alterations. Endocrinology 2008 149 1350-1357. (doi:10.1210/en.2007-1312)

16 Denhardt DT, Noda M, O'Regan AW, Pavlin D \& Berman JS. Osteopontin as a means to cope with environmental insults: regulation of inflammation, tissue remodeling, and cell survival. Journal of Clinical Investigation 2001107 1055-1061. (doi:10.1172/JCI12980)

17 Nomiyama T, Perez-Tilve D, Ogawa D, Gizard F, Zhao Y, Heywood EB, Jones KL, Kawamori R, Cassis LA, Tschöp MH et al. Osteopontin mediates obesity-induced adipose tissue macrophage infiltration and insulin resistance in mice. Journal of Clinical Investigation $2007 \mathbf{1 1 7}$ 2877-2888. (doi:10.1172/JCI31986)

18 Zeyda M, Gollinger K, Todoric J, Kiefer FW, Keck M, Aszmann O, Prager G, Zlabinger GJ, Petzelbauer P \& Stulnig TM. Osteopontin is an activator of human adipose tissue macrophages and directly affects adipocyte function. Endocrinology 2011152 2219-2227. (doi:10.1210/ en.2010-1328)

19 Ohmori R, Momiyama Y, Taniguchi H, Takahashi R, Kusuhara M, Nakamura H \& Ohsuzu F. Plasma osteopontin levels are associated with the presence and extent of coronary artery disease. Atherosclerosis 2003 170 333-337. (doi:10.1016/S0021-9150(03)00298-3)

20 Daniele G, Guardado Mendoza R, Winnier D, Fiorentino TV, Pengou Z, Cornell J, Andreozzi F, Jenkinson C, Cersosimo E, Federici M et al. The inflammatory status score including IL-6, TNF- $\alpha$, osteopontin, fractalkine, MCP-1 and adiponectin underlies whole-body insulin resistance and hyperglycemia in type 2 diabetes mellitus. Acta Diabetologia 201451 123-131. (doi:10.1007/s00592-013-0543-1)

21 Yan X, Sano M, Lu L, Wang W, Zhang Q, Zhang R, Wang L, Chen Q, Fukuda K \& Shen W. Plasma concentrations of osteopontin, but not thrombin-cleaved osteopontin, are associated with the presence and severity of nephropathy and coronary artery disease in patients with type 2 diabetes mellitus. Cardiovascular Diabetology 2010970. (doi:10.1186/1475-2840-9-70)
22 Kuwabara Y, Katayama A, Tomiyama R, Piao H, Kurihara S, Ono S, Mine K, Akira S, Orimo H \& Takeshita T. Gonadotropin regulation and role of ovarian osteopontin in the periovulatory period. Journal of Endocrinology 2015224 49-59. (doi:10.1530/JOE-14-0203)

23 Ahrens K, Mumford SL, Schliep KC, Kissell KA, Perkins NJ, WactawskiWende J \& Schisterman EF. Serum leptin levels and reproductive function during the menstrual cycle. American Journal of Obstetrics and Gynecology 2014210 248.e1-248.e9. (doi:10.1016/j.ajog.2013.11.009)

24 Asimakopoulos B, Milousis A, Gioka T, Kabouromiti G, Gianisslis G, Troussa A, Simopoulou M, Katergari S, Tripsianis G \& Nikolettos N. Serum pattern of circulating adipokines throughout the physiological menstrual cycle. Endocrine Journal 200956 425-433. (doi:10.1507/ endocrj.K08E-222)

25 Cella F, Giordano G \& Cordera R. Serum leptin concentrations during the menstrual cycle in normal-weight women: effects of an oral triphasic estrogen-progestin medication. European Journal of Endocrinology 2000142 174-178. (doi:10.1530/eje.0.1420174)

26 Hull MG, Savage PE, Bromham DR, Ismail AA \& Morris AF. The value of a single serum progesterone measurement in the midluteal phase as a criterion of a potentially fertile cycle ("ovulation") derived form treated and untreated conception cycles. Fertility and Sterility 198237 355-360.

27 Tarlatzis R, Fauser B, Chang J \& Azziz R. Revised 2003 consensus on diagnostic criteria and long-term health risks related to polycystic ovary syndrome (PCOS). The Rotterdam ESHRE/ASRM. Human Reproduction 200419 41-47. (doi:10.1093/humrep/deh098)

28 Ferriman D \& Gallwey JD. Clinical assessment of body hair growth in women. Journal of Clinical Endocrinology and Metabolism 196121 1440-1447. (doi:10.1210/jcem-21-11-1440)

29 Matthews DR, Hosker JP, Rudenski AS, Naylor BA, Treacher DF \& Turner RC. Homeostasis model assessment: insulin resistance and $\beta$-cell function from fasting plasma glucose and insulin concentrations in man. Diabetologia 198528 412-419. (doi:10.1007/BF00280883)

30 Geloneze B, Repetto EM, Geloneze SR, Tambascia MA \& Ermetice MN. The threshold value for insulin resistance (HOMA-IR) in an admixtured population. IR in the Brazilian Metabolic Syndrome Study. Diabetes Research and Clinical Practice 200672 219-220. (doi:10.1016/j.diabres. 2005.10.017)

31 Winhofer Y, Kiefer FW, Handisurya A, Tura A, Klein K, Schneider B, Marculescu R, Wagner OF, Pacini G, Luger A et al. CTX (crosslaps) rather than osteopontin is associated with disturbed glucose metabolism in gestational diabetes. PLOS ONE 20127 e40947. (doi:10.1371/journal. pone.0040947)

32 Saucedo R, Rico G, Vega G, Basurto L, Cordova L, Galvan R, Hernandez M, Puello E \& Zarate A. Osteocalcin, under-carboxylated osteocalcin and osteopontin are not associated with gestational diabetes mellitus but are inversely associated with leptin in non-diabetic women. Journal of Endocrinological Investigation 201538 519-526. (doi:10.1007/s40618-014-0220-4)

33 Liu LF, Kodama K, Wei K, Tolentino LL, Choi O, Engleman EG, Butte AJ \& McLaughlin T. The receptor CD44 is associated with systemic insulin resistance and proinflammatory macrophages in human adipose tissue. Diabetologia 201558 1579-1586. (doi:10.1007/s00125-015-3603-y)

Received 1 November 2015

Revised version received 21 December 2015

Accepted 23 December 2015 